\title{
A Novel Molecularly Imprinted Electrochemical Sensor Based on Dual Sensitization of MOF/CNTs and Prussian Blue for Detection of $17 \beta$-estradiol in Water
}

\author{
Dingding Duan ${ }^{1}$, Yaping Ding ${ }^{1}$, Li Li ${ }^{1}$, Lu Zhang ${ }^{1}$, Bingyu Jian ${ }^{1}$, Mingna Tan ${ }^{1}$, Yijing Huang ${ }^{1}$ \\ ${ }^{1}$ College of Science, Shanghai University, Shangda Road, Baoshan District, Shanghai 200444, P.R.C, \\ Corresponding e-mail address: wdingyp@sina.com, lilidu@shu.edu.cn
}

\begin{abstract}
:
In this paper, a novel molecular imprinted electrochemical sensor (MIECS) for 17ß-estradiol (E2) had been constructed. We synthesized MIL-53/CNTs hybrid to modify the glass carbon electrode (GCE) and used Prussian blue (PB) as electrocatalyst to accelerate electron transfer and increase surface area. Then, pyrrole and E2 were used as functional monomers and template respectively to form an imprinted film by electropolymerization. After removal of E2, the obtained molecularly imprinted sensor had been successfully used for detection of trace E2 in water. Under optimal conditions, the MIECS exhibited an excellent sensitivity and high selectivity. Moreover, a wide linear response range was obtained between $10^{-14}$ to $10^{-9}$, with an estimated detection limit of $6.19 \times 10^{-15} \mathrm{~mol} \mathrm{~L}^{-1}$. It was more sensitive than most E2 detection methods that had been reported in the past.
\end{abstract}

Key words: molecularly imprinted electrochemical sensor, prussian blue, MIL-53, polypyrrole, $17 \beta-$ estradiol

\section{Introduction}

$17 \beta$-estradiol (E2), one of the EDCs, can cause many negative effects in the endocrine system of humans and wildlife animals even at low concentration [1]. Due to the high activity of E2, a highly specific and sensitive method for determination of trace level of E2 is called for. Conventionally, E2 has been detected by various methods, such as high performance liquid chromatography (HPLC) [2], Gas Chromatography combined with Mass Spectrometry (GC/MS) [3], Capillary Electrophoresis [4], spectrophotometry [5] and enzyme-linked immune sorbent assay (ELISA) [6]. However, these detection techniques require tedious procedures and suffer from costly equipment. Thus, it is necessary to develop an effective method for fast, real-time and specific detection of trace E2 in practical applications.

In this work, we synthesized MIL-53/CNTs hybrid to modify the glass carbon electrode (GCE) and used PB as electrocatalyst to accelerate electron transfer and increase surface area. Then, polypyrrole (PPy) and E2 were used as functional monomers and template respectively to form a conductive imprinted film. The obtained molecularly imprinted sensor had been successfully used for detection of trace E2 in water.

\section{Characterization of MIP-PB-MIL/CNTs/GCE}

Fig. 1 is the morphology and microstructure of different modified materials investigated by TEM and SEM. Fig.1 (a) reveals that the MIL53 is assembled by the apparent crystalline structure with a size of about $50 \mathrm{~nm}$. As can be seen from Fig.1 (b), the mixture of MIL nanoparticles and CNTs formed conductive networks, increasing the specific surface area of the MIECS. Fig.1 (c) clearly shows that the size of nanoparticles was increased, indicating that PB nanocubes were successfully combined with MIL-53 nanoparticles. After electropolymerization, owing to the formation of the MIP film, the surface of conductive networks became rough and the size of nanoparticles increased further in Fig.1 (d). 


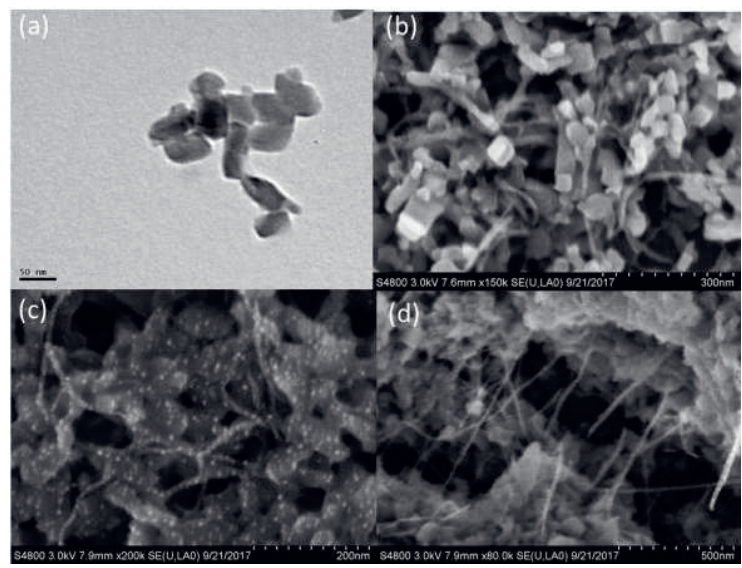

Fig.1. TEM images of (a) MIL-53 and SEM images of (b MIL-53/CNTs-GCE, (c) PB-MIL-53/CNTs-GCE (d) MIP-PB-MIL-53/CNTS-GCE.

Fig. 2 shows the electrochemical impedance spectroscopy of different modified electrodes. It can be seen clearly that the semicircle diameter gradually reduced through the layers of modification, indicating that the conductive performance of the modified GCE became better and better. Conversely, the increase of semicircle diameter indicates that the conductive PPy film was overoxidized and the templates were successfully eluted.

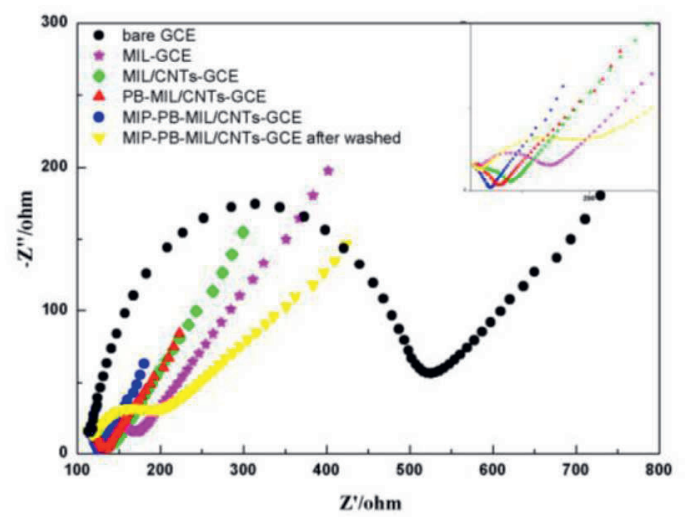

Fig.2. Electrochemical impedance spectra of different modified electrodes.

\section{Experimental result}

As can be seen in Fig.3, under the optimization of experimental conditions, the peak currents of MIP-PB-MIL/CNTs-GCE after rebinding 17ß-E2 are linear with the concentration of $17 \beta-E 2$ ranging from $10^{-14}$ to $10^{-9} \mathrm{~mol} \mathrm{~L}^{-1}$, which the linear relationship equation is $I_{p}(\mu A)=140.581+$ $4.32 \mathrm{lgC}\left(\mathrm{mol} \mathrm{L}^{-1}\right)\left(\mathrm{R}^{2}=0.993\right)$ with a detection limit of $6.19 \times 10^{-15} \mathrm{~mol} \mathrm{~L}^{-1}(\mathrm{~S} / \mathrm{N}=3)$. While the NIP-PB-MIL/CNTs-GCE without specific imprinted sites showed irregular current response.
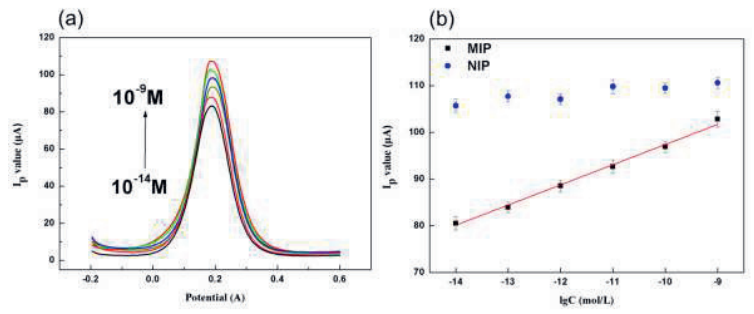

Fig.3. (a) DPV of MIP-PB-MIL/CNTS-GCE in a solution containing $5.0 \mathrm{mM} \mathrm{Fe}(\mathrm{CN})_{6}{ }^{3-4-}$ and $0.1 \mathrm{M}$ $\mathrm{KCl}$ after incubated in different concentrations of E2 in PBS (pH 3.0) for $8 \mathrm{~min}$. (b) Peak values of MIP$P B-M I L / C N T S-G C E$ and MIP-PB-MIL/CNTS-GCE versus the concentration of $E 2$.

This work is supported by the National Natural Science Foundation of China (No. 21671132)

\section{References}

[1] D. Wang, Z. Luo, X. Zhang, L. Lin, M. Du, G. Du Laing, C. Yan, Occurrence, Distribution and Risk Assessment of Estrogenic Compounds for Three Source Water Types in Ningbo City, China, Environmental Earth Sciences 74, 5961-5969 (2015); doi: 10.1007/s12665-015-4619-9

[2] L. Novakova, P. Solich, L. Matysova, J. Sicha, HPLC Determination of Estradiol, Its Degradation Product, and Preservatives in New Topical Formulation Estrogel HBF, Analytical and Bioanalytical Chemistry 379, 781-787 (2004); doi: 10.1007/s00216-004-2532-2

[3] B. YILMAZ, Simultaneous Determination of Estradiol Valerate and Medroxyprogesterone Acetate in a Tablet Formulation by Gas Chromatography-Mass Spectrometry, Analytical Sciences 26, 391-393 (2010); doi: 10.2116/analsci.26.391

[4] F. Regan, A. Moran, B. Fogarty, E. Dempsey, Novel Modes of Capillary Electrophoresis for the Determination of Endocrine Disrupting Chemicals, Journal of Chromatography A 1014, 141-152 (2003); doi: 10.1016/s0021-9673(03)01036-7

[5] B. Yilmaz, Y. Kadioglu, Determination of $17 \beta-$ estradiol in Pharmaceutical Preparation by UV Spectrophotometry and High Performance Liquid Chromatography Methods, Arabian Journal of Chemistry 10, S1422-S1428 (2017); doi: 10.1016/j.arabjc.2013.04.018

[6] C.P. Silva, D.L. Lima, R.J. Schneider, M. Otero, V.I. Esteves, Development of ELISA Methodologies for the Direct Determination of 17beta-estradiol and 17alpha-ethinylestradiol in Complex Aqueous Matrices, Journal of Environmental Management 124, 121-127 (2013); doi: 10.1016/j.jenvman.2013.03.041 